\title{
(Des)metaforizando significados na pós-edição: um estudo exploratório do esforço despendido por estudantes de tradução
}

(De)metaphorizing meanings in postediting: an exploratory study on the effort spent by translation students

\author{
Adriana Silvina Pagano \\ Universidade Federal de Minas Gerais \\ Cristiano Gonçalves Araújo' \\ Universidade Federal de Minas Gerais
}

\section{Resumo}

Este artigo examina dados de um experimento-piloto e tem por objetivo investigar o esforço despendido por estudantes de tradução em uma tarefa de pós-edição, a partir da análise de pausas e movimentos recursivos registrados durante a produção textual em tempo real para instâncias gramaticalmente metafóricas ( $c f$. HALLIDAY, 1998). O estudo contempla o processo e o produto da pósedição de três alunos de pós-graduação em tradução. Para se proceder à análise, foram observados os registros de uso de teclado e movimentos de cursor mapeados pelo programa Translog 2006 para a produção textual em tempo real. Como resultado, verificou-se um grande esforço para se processar algumas instâncias gramaticalmente metafóricas, sendo que esse esforço não resultou em uma maior adequação dos significados produzidos.

\section{Palavras-chave}

pós-edição; produção textual em tempo real; metáfora gramatical; 
esforço.

\section{Abstract}

This paper reports on data of a pilot study and aims at analysing the effort spent by translation students in a postediting task based on pauses and recursiveness when dealing with instances of grammatical metaphorization ( $c f$. HALLIDAY, 1998) during ongoing text production. The study analyses the process and the end product of a postediting task carried out by three graduate translation students, drawing on keylogging data recorded by the software Translog 2006. Results show that some instances of grammatical metaphorization were more effortful to the translator, although effort did not necessarily result in the construal of more adequate meanings.

\section{Keywords}

postediting; ongoing text production; grammatical metaphor; effort. 


\section{Introdução}

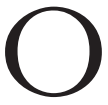

escasso diálogo entre as abordagens e teorias desenvolvidas no escopo dos Estudos da Tradução e as aplicações da Tradução Automática (doravante TA) é apontado por diferentes autores (e.g. KOBY, 2001; MATTHIESSEN, 2001; GASPARI, 2007). Essa constatação suscita reflexões, pois, conforme ressalta Koby (2001), esse diálogo poderia ser bastante profícuo, em especial, no que concerne às aplicações da TA que envolvam a participação humana, como a pós-edição de textos traduzidos automaticamente.

A pós-edição é assinalada desde os primeiros trabalhos sobre a TA como condição necessária para que textos traduzidos automaticamente atinjam um nível de qualidade aceitável ( $c f$. BAR-HILLEL, 1960); entretanto, passado mais de meio século desde as primeiras reflexões sobre o tema, pouco ainda se sabe sobre o processo e produto dessa tarefa ( $c f$. KRINGS, 2001; KOBY, 2001; ALMEIDA e O`BRIEN, 2010). Esse cenário deve-se, por um lado, ao fato de a TA almejar prescindir da intervenção humana no processo de tradução, o que resulta em investimentos de pesquisa direcionados ao aprimoramento de sistemas automáticos; por outro lado, ao fato de os Estudos da Tradução comumente não buscarem teorizar a complementaridade entre a tradução humana e a automática ( $c f$. MATTHIESSEN, 2001). É certo, contudo, que a carência de estudos sobre a pós-edição aponta para um descompasso entre a teoria e a prática, haja vista que, conforme aponta Allen (2003), há no mercado de tradução uma crescente demanda pelo uso de sistemas automáticos de tradução e pós-edição.

$\mathrm{Na}$ literatura, verifica-se que os primeiros trabalhos sistemáticos sobre a pós-edição, desenvolvidos no escopo dos Estudos da Tradução, começaram a surgir a partir da década de 1980, orientados ao produto e à identificação de 
competências (e.g. WAGNER, 1983; LOFFLER-LAURIAN, 1984; VASCONCELLOS, 1986a; VASCONCELLOS, 1989). Na década de 1990, Krings (2001) amplia o conhecimento sobre a pós-edição, ao investigar de forma pioneira os aspectos processuais nela envolvidos. Já a partir de 2000, um crescente número de estudos vem investigando a pós-edição ( $c f$. ALLEN, 2003; O`BRIEN, 2002, 2006; RAMOS, 2010; CARL et al, 2011), notando-se um maior enfoque no processo e na descrição de competências, embora questões linguísticas e funcionais também sejam consideradas. Esses estudos contribuíram para um melhor entendimento de aspectos linguísticos, funcionais e processuais da pós-edição, valendo-se de métodos e abordagens já tradicionalmente consolidados nos Estudo da Tradução. Contudo, como a grade maioria desses trabalhos aponta, muito há a ser desenvolvido.

A fim de contribuir com os estudos sobre a pós-edição, este trabalho se baseia em dados de um experimento-piloto e tem por objetivo a descrição de processos de (des)metaforização gramatical em instâncias que demandam esforço para serem processadas, a partir da observação de pausas e movimentos recursivos de produção textual. No escopo desta proposta, é apresentada uma análise exploratória, em que se descreve o processo logogenético de produção textual, identificando-se Unidades de Tradução e Unidades de Alinhamento (cf. ALVES et al, 2010). A partir dessa descrição, é proposta uma discussão sobre o esforço, o processo de desmetaforização e os significados produzidos.

\section{A delimitação do estudo}

Este trabalho propõe-se a adotar a tradição pautada pelos métodos e insumos utilizados nos Estudos da Tradução, a fim de melhor entender a participação humana na pós-edição. Para tanto, busca promover um diálogo ainda pouco difundido nos Estudos da Tradução, de se abordar o produto e o proces-so de produção de significados de forma mais integrada, como já explorado em alguns estudos ( $c f$. ALVES et al, 2010; ALVES et al, 2011).

O enfoque no produto parte de uma tradição de pesquisas em pósedição voltada à identificação de modificações linguísticas implementadas na tradução automática crua (cf. ALMEIDA e O`BRIEN, 2010; O`BRIEN, 2006; VAS- 
CONCELLOS, 1989), as quais se sustentam na afirmação de que um estudo linguístico das intervenções realizadas no produto da Tradução Automática, em diferentes níveis de refinamento, pode nos ajudar a priorizar nossas estratégias. Ao estratificarmos os tipos de correções implementadas, podemos começar a orientar a nossa forma de tratar a pós-edição, de forma que os sistemas atuais de TA sejam utilizados de forma mais efetiva (VASCONCELLOS, 1989, p.89). ${ }^{2}$

Essa reflexão, datada do final da década de 1980, parece ainda ser válida nos dias atuais, quando os sistemas de TA, significativamente mais refinados, ainda não possibilitam a geração de uma tradução automática e de alta qualidade de todo e qualquer texto ( $c f$. MARTINS, 2008).

Conforme mencionado, o interesse pela descrição das modificações implementadas pelo pós-editor constitui um foco tradicional de estudos sobre a pós-edição. Vansconcellos (1989), por exemplo, estuda as mudanças implementadas na pós-edição enfocando contrastes tipológicos entre o inglês e o espanhol. O`Brien (2006), por sua vez, estuda a correlação entre esforço e alguns indicadores de traduzibilidade, enfocando o impacto da estandardização da linguagem em relação às mudanças implementadas pelo pós-editor.

De acordo com O`Brien (2004; 2006), os indicadores negativos de traduzibilidade correspondem a estruturas linguísticas reconhecidamente problemáticas para os sistemas de TA, como o uso de voz passiva, de grupos nominais extensos, de elipses e de orações não-finitas. uma vez sendo um problema para os sistemas de TA, também são potenciais obstáculos para o pós-editor, demandando um maior esforço para serem interpretados e modificados de acordo com os padrões e normas da língua-alvo.

Esse conflito entre as demandas funcionais e sintáticas na pós-edição é também apontado por Vasconcellos (1986a; 1986b), que observa a necessidade de se reconfigurarem os elementos da oração, durante o processo de pós-edição. Ademais, Vasconcellos (1986a; 1986b) aponta para a necessidade de se relacionarem essas demandas a princípios linguísticos mais amplos, a fim de que os resultados possam ter maior significância tanto para a TA quanto para os ti- 
pos de tradução que envolvem a intervenção humana. No caso de Vasconcellos (1986a; 1986b; 1989), ela remete suas observações aos princípios linguísticos da teoria sistêmico-funcional da linguagem ( $c f$. HALLIDAY e MATTHIESSEN, 2004).

Seguindo a proposta de Vasconcellos (1986a; 1986b) de remeter as demandas da pós-edição a princípios linguísticos teoricamente fundamentados, este trabalho adota a teoria sistêmico-funcional de linguagem como parte integrante de sua fundamentação teórica e descritiva, explorando, no escopo desta teoria, o conceito de metáfora gramatical (cf. HALLIDAY, e MATTHIESSEN, 1999). Assim, além de potencializar a descrição e análise, busca-se evitar o uso de categorizações linguísticas teoricamente difusas e arbitrárias, como as empregadas por Almeida e O`Brien (2010), ao utilizarem uma adaptação do modelo LISA QA (The Localization Industry Standards Association - Quality assessment) e GALE (Post-editing guidelines) na descrição das modificações feitas no processo de pós-edição.

A metaforização gramatical é um conceito da teoria sistêmico funcional retomado por Steiner $(2001 ; 2005)$ no estudo das propriedades dos textos traduzidos. Steiner (2001) aponta que a descrição dessas propriedades deve levar em consideração a tipologia linguística das línguas envolvidas, a comparação de registros e o processo de tradução. No caso do processo de tradução, esse autor afirma haver uma série de fatores que devem ser considerados, mas seu modelo enfoca especificamente um, qual seja, o entendimento (understanding) do texto-fonte. Contudo, para trabalhar com esse fator na perspectiva sócio-semiótica que adota, Steiner estabelece uma correlação entre o entendimento e evidências linguísticas características do processo de (des)metaforização gramatical (grammatical (de)metaphorization). Assim, modela o entendimento do texto traduzido a partir de evidências linguísticas e, ainda, estabelece uma relação entre o processo de desmetaforização e a propriedade de explicitação em textos traduzidos.

Partindo do trabalho de Steiner (2005) e de sua hipótese de desmetaforização gramatical, Alves et al (2011) apresentam um desdobramento promissor, ao enfocar não somente o produto final da tradução, mas o processo logogenético de produção de significados em instâncias que demandam esforço e envolvem processos de (des)metaforização. É no escopo desta proposta que este es- 
tudo se contextualiza, enfocando a pós-edição.

\section{O estudo da pós-edição}

Segundo Hutchins (2006), o termo pós-edição é utilizado em diferentes campos do processamento de linguagem natural. Ao ser empregado no contexto da TA, refere-se à tarefa de correção do texto traduzido pela máquina, a qual é comumente desempenhada por um linguista, tradutor ou pós-editor ( $c f$. ALLEN, 2003; KOBY, 2001). Assim, a pós-edição insere-se como um ramo da Tradução Automática Auxiliada por Humano.

A concepção de se pós-editar o texto de saída, como forma de se adequá-lo a propósitos específicos, já figurava nos primeiros trabalhos sistemáticos sobre TA ( $c f$. BAR-HILLEL, 1960), que viam na pós-edição um meio de se corrigir os erros produzidos pelos sistemas de TA, uma vez que a produção de traduções automáticas de alta qualidade era uma realidade distante. Nesse contexto, é importante mencionar que a pós-edição, por um lado, era uma necessidade, devido às limitações dos sistemas de TA. Por outro lado, entretanto, era tida como problemática, devido ao retardo no acesso à informação. Dessa forma, com o objetivo de otimizar o acesso a informações em diferentes línguas, muitas pesquisas foram e continuam sendo desenvolvidas a fim de se ampliar o poderio dos sistemas de TA e, com isso, diminuir ou eliminar a necessidade da pós-edição realizada por humanos ( $c f$. KOBY, 2001). Contudo, o efeito dessas pesquisas, que resultaram em melhorias nos sistemas de TA, ao invés de reduzir, serviu a um aumento significativo da demanda por textos pós-editados ( $c f$. ALLEN, 2003).

Ao se considerar o estudo sistemático da pós-edição, verifica-se haver um grande enfoque em aspectos técnicos relacionados a sua aplicabilidade ( $c f$. RAMOS, 2010; MARTÍNEZ, 2003; ALLEN, 2003), pautando-se pela asserção de que

Em uma era em que os sistemas de TA já são capazes de traduzir milhões de palavras por hora, o tempo que o sistema leva para gerar a tradução se torna insignificante. Assim, o custo-benefício do sistema de TA deve ser mensurado é pela efetividade do processo de pós-edição. (RYAN apud O`BRIEN, 2002, p. 100)3 
De fato, o estudo sobre a efetividade do processo de pós-edição tem trazido importantes contribuições para o seu entendimento, em especial quando abordado a partir dos subsídios metodológicos e teóricos empregados nos Estudos da Tradução. Nesse escopo, verifica-se haver estudos direcionados ao produto, ao processo e às competências envolvidas na pós-edição.

Em relação ao estudo do produto da pós-edição, sobressaem-se os trabalhos de Vasconcellos (1986a; 1986b; 1989), que, enfocando diferenças tipológicas entre o inglês e o espanhol, apresentam dados visando a uma otimização do processo de pós-edição nesse par linguístico. Menciona-se, também, o trabalho de doutorado de O`Brien (2006), que investiga o impacto da estandardização da linguagem em uma tarefa de pós-edição.

Em relação ao estudo do processo de pós-edição, sobressai-se o trabalho de Krings (2001), possivelmente a obra mais abrangente já publicada sobre o tema. Em seu estudo de caráter descritivo, Krings (2001) enfoca o processo por meio da análise do esforço temporal, técnico e cognitivo dispendido em uma tarefa de pós-edição, investigando, também, a influência de se pós-editar com ou sem acesso ao texto-fonte. Como conclusão, o autor aponta sete tipos de processos envolvidos na pós-edição, que se correlacionam a questões textuais, a habilidades motoras e estratégicas e ao monitoramento cognitivo da tarefa.

Já em relação ao estudo das competências em pós-edição, ressaltam-se os trabalhos de Wagner (1983), Vasconcellos (1986a) e Almeida e O`Brien (2010). O estudo de Wagner (1983) enfoca as competências para se proceder a uma pós-edição rápida e de menor qualidade, e aponta que

um pouco de confiança na própria habilidade de tradução e certa expertise técnica são essenciais para este tipo de trabalho. Embora a pós-edição rápida produza uma tradução de menor qualidade, não se deve pensar que ela pode ser realizada por profissionais inexperientes. Pelo contrário - ao menos que o pós-editor tenha um alto nível de conhecimento linguístico e técnico, ele não será capaz de pós-editar a tradução automática crua atingindo um padrão razoável de qualidade no tempo prescrito. (WAGNER, 1983, p.204) ${ }^{4}$

Dessa forma, Wagner (1983) descreve que possuir (a) um excelente co- 
nhecimento das línguas fonte e alvo, (b) conhecimento do assunto do texto, (c) experiência com processadores de texto e (d) tolerância ao uso de tradutores automáticos são características necessárias a um pós-editor.

A essa relação de competências, Vasconcellos (1986a) acrescenta que é necessário possuir (a) conhecimentos sobre tradução automática (isto é, sobre o que ocorre dentro da caixa-preta, o motivo de certos erros ocorrerem de forma consistente, e as limitações e potencialidades dos sistemas), (b) habilidades para gerenciar banco de dados terminológicos, (c) conhecimentos sobre pré-edição e estandardização da linguagem, (d) capacidade de programar macros nos processadores de texto, e (e) sólidos conhecimentos sobre linguística textual.

Já Almeida e O`Brien (2010) descrevem ainda ser necessária (a) uma habilidade de identificação dos itens da tradução automática crua que devem ser modificados (isto é, identificar as mudanças essenciais), (b) uma habilidade de se pós-editar com rapidez (cerca de 5.000 palavras ao dia), e (c) uma capacidade de seguir as orientações da tarefa de pós-edição, a fim de se reduzir o número de intervenções estilísticas e de cunho pessoal.

Diante dessas muitas demandas e competências, verifica-se que a pósedição é um trabalho bastante especializado, razão pela qual grande parte dos autores sugere que ela seja realizada por tradutores profissionais, os quais, idealmente, devem ser treinados e possuírem alguma experiência, haja vista que as habilidades para se pós-editar um texto são desenvolvidas gradualmente ( $c f$. VASCONCELLOS, 1989; KOBY, 2001; O, BRIEN, 2002).

Assim, embora alguns tradutores tenham uma atitude hostil em relação ao uso de programas de tradução automática, seja por (a) medo de demonstrar menor proficiência linguística, por (b) medo de ter a liberdade de expressão limitada, ou por (c) negar-se a corrigir erros repetitivos, as habilidades que possuem para identificar erros contrastivos, seus conhecimentos técnicos de informática e sua base de conhecimento sobre tradução fazem desses profissionais os mais adequados para realizarem tarefas de pós-edição (cf. O`BRIEN, 2002).

Logo, sendo a pós-edição e a tradução tarefas correlatas, embora não idênticas do ponto de vista cognitivo ou de suas demandas linguísticas ( $c f$. KRINGS, 
2001; O'BRIEN, 2002), verifica-se que "muitas das características atribuídas a bons tradutores também se aplicam a bons pós-editores" ( $c f$. OFFERSGAARD et al apud ALMEIDA e O`BRIEN, 2010, s.p). Entretanto, "há uma importante habilidade que é específica à pós-edição: a habilidade de decidir rapidamente se um segmento de uma tradução automática deve ser ou não usado" (ibid).5

É importante ressaltar, contudo, que grande parte destas considerações sobre as competências em pós-edição valem-se de insumos providos por estudos sobre o produto e o processo desta tarefa.

Almeida e O`Brien (2010), por exemplo, sugerem que a experiência prévia em tradução pode ter um impacto maior na tarefa de pós-edição do que uma experiência prévia em pós-edição. De igual modo, descrevem que tradutores experientes tendem a implementar um maior número de mudanças estilísticas, por serem treinados a polir os textos, e ainda assim serem mais eficientes na realização da tarefa de pós-edição. Nesse caso, afastam-se do protocolo da pós-edição de efetuar um número mínimo de intervenções.

Essas considerações apresentadas por Almeida e O`Brien (2010) baseiam-se em um estudo descritivo do produto e processo da pós-edição, enfocando a experiência prévia em tradução. Assim, contribuem para um melhor entendimento das competências e habilidades necessárias para se pós-editar textos, além de levantar dados que contribuem para um melhor entendimento sobre a natureza desta tarefa.

De igual forma, este estudo exploratório busca contribuir com a efetividade do trabalho de pós-edição, ao ampliar o entendimento de como se dá a intervenção humana nesse processo de produção de significados, nesse caso, informada pelos conceitos de metáfora gramatical, e de unidades de tradução e de alinhamento, os quais serão retomados a seguir.

\section{Metáfora gramatical}

A metáfora gramatical é um conceito da linguística sistêmico-funcional e, em uma definição simplificada, pode ser entendida como formas diferentes de se dizer a "mesma coisa" (HALLIDAY e MATTHIESSEN, 1999). Contudo, é so- 
mente com referência ao escopo mais amplo da teoria sistêmico funcional que esse uso da linguagem pode ser mais bem compreendido, razão pela qual cabe retomar alguns conceitos dessa teoria.

Na linguística sistêmico-funcional, entende-se que a gramática de uma língua natural é uma teoria da experiência humana, ou seja, é o que transforma a experiência humana em significado e permite a performance de relações interpessoais, sendo que essas duas funções se relacionam a uma terceira, que possibilita a criação de discursos. Essa configuração de significados compõe a hipótese metafuncional da teoria sistêmica, que pode ser sistematizada da seguinte forma:

(meta)Função:

(a) ideacional (função reflexiva) representar a experiência interna e externa ao indivíduo

(b) interpessoal (função de ação) desempenhar papéis sociais

(c) textual (função discursiva) organizar a linguagem em discurso

Embora não haja uma relação de proeminência hierárquica entre essas metafunções da linguagem, haja vista que todas atuam simultaneamente na construção dos significados, a metáfora gramatical, da forma em que é retomada neste trabalho, é tratada mais detidamente a partir de seu viés ideacional, ou seja, ela é retomada a partir do enfoque em como os sujeitos constroem semioticamente representações da experiência.

Como teoria da experiência humana, a gramática ideacional possibilita a categorização da experiência e a imposição de ordem à forma como fenômenos se relacionam. Contudo, cabe ressaltar que não se trata de categorizar ou impor ordem a uma realidade pré-existente, em um processo passivo de correspondência entre um mundo material dado e um ordenamento semiótico construído. Enquanto teoria da experiência humana, a gramática não apenas reflete ou codifica eventos naturais dados. Ela constitui de forma não arbitrária o próprio mundo material enquanto significado (HALLIDAY e MATTHIESSEN, 1999).

Conforme descrevem Halliday e Matthiessen (1999), a gramática ideacional constrói a experiência enquanto processo, na forma de uma unidade gra- 
matical, ou oração. Cada processo, por sua vez, é construído em uma estrutura gramatical, cuja configuração é composta pelo (i) próprio processo, (ii) por entidades que participam do processo e (iii) por elementos circunstanciais que se associam ao processo; esses elementos são, por sua vez, representados por classes gramaticais como verbos, substantivos, advérbios etc.

Nesse escopo, verifica-se que um dos pontos mais importantes é a observação de que o mesmo processo evolucionário que permite a construção da experiência, transformando-a em significado, também permite que a experiência, uma vez construída enquanto significado, seja reconstruída de outras formas pela gramática, o que é possível porque o plano de conteúdo da língua é estratificado em dois níveis, o semântico e o lexicogramatical. Dessa forma, a lexicogramática constrói uma representação semântica da experiência, e uma vez construída essa representação, ela pode ser reconstruída de diferentes formas na lexicogramática.

A metáfora gramatical se identifica com a reconstrução da experiência a partir de um significado realizado de forma congruente. Assim, em uma perspectiva semiogenética, pode se dizer que a experiência é construída em um primeiro momento como uma realização lexicogramatical congruente no estrato semântico, a qual, uma vez realizada como significado, pode ser reconfigurada de diferentes formas no estrato lexicogramatical, sendo essas diferentes formas síndromes de processos de metaforização gramatical, os quais se caracterizam estruturalmente pela mudança de ordem (rank shift), pela mudança de classe gramatical, e pela transcategorização (HALLIDAY e MATTHIESSEN, 1999).

A escala de ordem se refere à forma como a experiência é organizada estruturalmente, se em complexos oracionais, orações, grupos ou frases preposicionadas. No caso em tela, interessa saber que o que é realizado congruentemente em ordens superiores (e.g. complexo oracional e oração), em versões mais metafóricas, tende a ser realizado como grupos ou frases preposicionadas, por exemplo.

Em uma sistematização do que se entende por metáfora gramatical, em seu viés ideacional, Teich (2001, p. 227) aponta que:

$\mathrm{Na}$ teoria sistêmico-funcional, o termo metáfora gramatical se relaciona ao 
que é comumente identificado como paráfrase, abrangendo, por exemplo, a nominalização de um processo. A ideia aqui subjacente é que há uma realização lexicogramatical congruente, de um determinado conteúdo experiencial (e.g. um processo semântico é realizado por uma oração, uma entidade semântica é realizada por um grupo nominal, etc.), e um número de realizações não congruentes, as quais diferem textual e interpessoalmente da realização congruente. ${ }^{6}$

A fim de exemplificar sua definição, Teich (2001, p. 227) apresenta os exemplos que são reproduzidos a seguir e aponta que, enquanto a forma congruente de expressão de um processo ou evento é comumente uma oração com modo oracional (exemplo 3), o mesmo conteúdo experiencial pode ser expresso de diferentes formas, em diferentes níveis de metaforicidade (exemplos 4, 5 e 6). Exemplos:

(3) The student objects to the theory

(4) The student's objecting to the theory

(5) The student's objection to the theory

(6) That the student objects to the theory

Conforme se observa nesses exemplos, a realização de metáforas gramaticais envolve mudança de classe gramatical (e.g. de verbo para nome) e mudança de ordem, o que, conforme apresentado, são características intrínsecas ao processo de metaforização gramatical (HALLIDAY e MATTHIESSEN, 2004). Assim, o que é instanciado de forma congruente no exemplo (3), na ordem da oração, é reconstruído com um crescente nível de metaforicidade em (4) e (5), na ordem do grupo nominal, e em (6), como determinante de um grupo nominal.

Ademais, a mudança de ordem comumente implica em uma reconfiguração funcional dos constituintes da oração, resultando em processos de transcategorização. Nesse caso, ao remapearem a experiência (em grupos nominais, por exemplo), as realizações metafóricas permitem a criação de uma progressão lógica necessária, por exemplo, ao discurso científico e burocrático. Outrossim, esse tipo de realização permite o estabelecimento de relações coesivas, por meio da criação de taxonomias, de repetições do conteúdo experiencial, mesmo que em diferentes níveis de metaforicidade, etc., além de possibilitar a pro- 
gressão do fluxo de informação, ao condensar (e.g. em um grupo nominal em posição temática) informações apresentadas anteriormente de forma mais congruente ( $c f$. HALLIDAY, 1998).

\section{A metáfora gramatical no estudo da tradução}

Conforme exposto, devido ao seu potencial de abordar a relação entre a semântica e a lexicogramática, a metáfora gramatical é retomada por Steiner (2001; 2005) no estudo das propriedades dos textos traduzidos. Steiner (2001) aponta que a descrição dessas propriedades deve levar em consideração a tipologia linguística das línguas envolvidas, a comparação de registros e o processo de tradução.

Ao abordar o processo de tradução, Steiner (2001) afirma haver uma série de fatores a serem considerados, mas seu modelo enfoca especificamente um, qual seja, o entendimento do texto-fonte. Contudo, para trabalhar com esse fator na perspectiva sócio-semiótica que adota, Steiner estabelece uma correlação entre o entendimento e evidências linguísticas características do processo de (des)metaforização gramatical. Assim, propõe uma modelagem do entendimento do texto traduzido a partir de evidências linguísticas e, ainda, estabelece uma relação entre o processo de metaforização e a propriedade de explicitação em textos traduzidos.

No escopo dessa proposta, conforme apontam Alves et al (2010), é possível identificar três possíveis fenômenos, nomeadamente:

(a) metaforização $\rightarrow$ o enunciado da língua-alvo é mais metafórico do que o da língua-fonte.

(b) desmetaforização $\rightarrow$ o enunciado da língua-fonte é mais metafórico do que o da língua-alvo.

(c) remetaforização $\rightarrow$ o mesmo nível de metaforicidade é encontrado em ambas as línguas.

Para se identificarem esses fenômenos na tradução, os membros do projeto CroCo, desenvolvido na Universidade do Sarre, Alemanha, e ao qual o trabalho de Steiner se vincula ( $c f$. HANSEN-SCHIRRA et al, 2007), propõem um 
mapeamento de segmentos do texto-fonte em relação a segmentos do textoalvo em multicamadas, isto é, considerando as partes do discurso (i.e. substantivos, verbos, adjetivos, etc.), a função gramatical (processo, participante, circunstância) e a escala de ordem (mapeamento de palavra, frase, grupo e oração). Portanto, consderam os elementos nos quais os processos de metaforização gramatical reverberam.

Dessa forma, esse mapeamento multicamadas, que envolve uma anotação linguisticamente informada e um alinhamento de segmentos, possibilita a identificação de como unidades semânticas são realizadas no estrato lexicogramatical, dos textos em relação tradutória. O mapeamento indica, assim, as mudanças (shifts) implementadas pelo tradutor e provê evidências linguísticas sobre seu processo de entendimento.

\section{Unidades de alinhamento e unidades de tradução}

O alinhamento de instâncias do produto de textos em relação tradutória possibilita a identificação de unidades de alinhamento ( $c f$. CARL, 2009), as quais podem ser estabelecidas a partir de diferentes critérios linguísticos, como a escala de ordem e a função gramatical ( $c f$. HANSEN-SCHIRRA et al 2007; ALVES et al, 2010). Assim, quando duas sentenças em relação tradutória não apresentam mudanças (isto é, são linguisticamente equivalentes), suas palavras, frases, grupos e funções gramaticais estarão em uma relação de alinhamento paralelo.

Contudo, conforme observam Alves et al (2010), a tradução comumente implica em mudanças em diferentes níveis?. Assim, embora seja possível alinhar um substantivo do texto-fonte com um substantivo do texto-alvo, pode ocorrer que estes estejam desempenhando diferentes funções gramaticais. Logo, tem-se um alinhamento de classe, mas não de função.

Ademais, conforme apontado pelo trabalho de Hansen-Schirra et al (2007), há dois fenômenos bastante comuns ao se abordar o alinhamento em tradução. O primeiro deles é identificado como links vazios (empty links) e refere-se àqueles segmentos que não possuem um correspondente nos textos fonte ou alvo. O outro fenômeno é identificado como linhas cruzadas (crossing lines) e refe- 
re-se àqueles segmentos do texto-fonte que só possuem um correspondente no texto-alvo em outro nível de análise. Por exemplo, quando um grupo nominal de um texto-fonte é realizado como um determinante de um grupo nominal de um texto-alvo, na mesma oração ou não, tem-se um caso de linha cruzada.

Menciona-se, contudo, que apesar dessas dificuldades inerentes ao processo de alinhamento, este possibilita a investigação de instâncias de metaforização gramatical, o que pode ser feito analisando-se o produto da tradução ( $c f$. HANSEN-SCHIRRA et al, 2007). Ressalta-se, porém, que para se ter acesso ao processo logogenético de produção textual e aos processos recursivos que levam o tradutor a certas escolhas, é necessário mapear as unidades de alinhamento em relação às unidades de tradução (cf. ALVES et al, 2011).

As unidades de tradução correspondem aos significados processados durante a atividade de tradução, sendo que a definição dessas unidades depende, sobremodo, da análise de pausas e do foco de atenção do tradutor ( $c f$. ALVES e VALE, 2009; 2011).

A análise de pausas é importante porque, segundo apontam Alves e Vale (2009), uma unidade de tradução se inicia com uma pausa registrada pelo monitoramento de uso do teclado, a partir da qual segue uma fase contínua de produção textual que se encerra com outra pausa. Cabendo mencionar que estas pausas podem advir de uma necessidade de se orientar, planejar ou fazer consultas durante a tarefa.

Por sua vez, o foco de atenção do tradutor interessa porque, conforme descrevem Alves e Vale (2009), em essência as unidades de tradução são segmentos do texto-fonte, de qualquer natureza ou extensão, que atraem o foco de atenção do tradutor em um dado momento do processo. Dessa forma, conforme observam Alves et al (2010, p. 123-124),

embora as unidades de tradução correspondam a segmentos do texto-fonte, é por meio da análise do processo de produção do texto-alvo que podemos momentaneamente mapeá-las, enquanto segmentos de produção textual do textoalvo, localizado entre pausas (intervalos não-produtivos), que pode ser alinhado a um segmento do texto-fonte. Assim, esses segmentos, embora não sejam propriamente as unidades de tradução, correlacionam-se a elas. Logo, 
um segmento é um excerto de texto passível de ser observado nos textosalvo ou no processo de produção textual, ao passo que as unidades de tradução são identificadas no tempo por intervalos de pausa que refletem o foco de atenção do tradutor. ${ }^{8}$

A partir dessa descrição, verifica-se que as unidades de tradução são indiretamente observadas, a partir da consideração de um segmento do textoalvo produzido em um intervalo de pausas.

Esses segmentos, por sua vez, podem ser retomados durante o processo pelo tradutor, para revisão ou somente orientação, em movimentos recursivos mais ou menos frequentes. Para abordar esses movimentos recursivos em relação às unidades de tradução, Alves e Vale (2009) propõem a distinção de dois níveis de unidades, que se correlacionam entre si, nomeadamente, as micro e macrounidades de tradução.

Por definição, tanto as micro quanto as macrounidades de tradução correspondem a segmentos de produção textual ( $c f$. ALVES e VALE, 2011), embora estejam correlacionadas ao foco de atenção do tradutor, sendo que, enquanto as microunidades correspondem ao fluxo de contínua produção textual entre um intervalo de pausas (cuja duração é dada de forma arbitrária), as macrounidades de tradução correspondem a todas as produções textuais propostas para um mesmo segmento do texto-fonte, mapeado a partir da identificação de uma microunidade inicial (ALVES e VALE, 2011). Assim, uma sequência de microunidades implementadas em um mesmo segmento (enquanto foco de atenção) compõe uma macrounidade de tradução.

É importante ressaltar que o mapeamento de micro e macrounidades de tradução permite a investigação de processos recursivos e a identificação de como ocorre processos de (des/re)metaforização gramatical (ALVES et al, 2011). Ademais, por se tratarem de segmentos de produção textual, podem ser analisados em relação às unidades de alinhamento e à luz de uma teoria linguística, razão pela qual são retomadas neste trabalho. 


\section{Metodologia}

Esta pesquisa toma como ponto de partida os dados de um experimento-piloto que contou com a participação de cinco estudantes de pós-graduação em Tradução, sendo que quatro realizaram a tarefa de pós-edição, e um realizou a tarefa de tradução.

Para esse experimento-piloto foi selecionado um excerto de um texto jornalístico originalmente escrito em inglês, e sua tradução automática crua, para o português, realizada pelos sistemas Google Tradutor e Systran (versão gratuita), reproduzidos no Anexo 1. Esse extrato possui 223 palavras e, embora não represente a notícia na íntegra, possui tessitura e coesão, realizando os significados ideacionais e interpessoais de forma coerente ao texto da íntegra.

O texto é veiculado pelo site de notícias e opinião The Daily Beast, o qual pertence ao grupo da revista norte-americana Newsweek. Escrito por Daniel Stone, e publicado em 30 de outubro de 2011, aborda aspectos da política norteamericana em relação à crise de gastos.

Considerando-se suas propriedades tipológicas ( $c f$. HERKE-COUCHMAN, 2006), verifica-se tratar-se de um texto monológico, no modo escrito, e que apresenta um predomínio do processo sócio-semiótico relatar. Com efeito, possui uma progressão temática característica, em que os temas são comumente retomados por coesão referencial, e uma progressão lógico-semântica que alterna ocorrências de projeção e expansão ( $c f$. HALLIDAY e MATTHIESSEN, 2004). Ademais, ressalta-se tratar-se de um texto que apresenta algumas dificuldades para serem processadas pelos sistemas de TA, referente a questões terminológicas (e.g. Tea Party), a ambiguidades (e.g. Pork), e a alguns indicadores negativos de traduzibilidade, como grupos nominais extensos e orações não-finitas.

Com essa configuração funcional, o texto apresenta algumas demandas para ser pós-editado, dentre as quais (a) um certo conhecimento do campo (isto é, do assunto) da notícia, (b) um reconhecimento de suas questões terminológicas e de suas ambiguidades, (c) e um certo conhecimento da configuração funcional de um relato em português. Em outras palavras, retomando-se a proposta de Steiner (2005), demanda (a) um reconhecimento de diferenças tipológicas 
entre os sistemas do inglês e do português, (b) um reconhecimento das diferenças referentes ao registro do texto nestes sistemas, e (c) um entendimento dos significados ideacionais, interpessoais e textuais nele realizado.

Por ocasião do experimento-piloto, os estudantes de tradução receberam a orientação de que não haveria pressão de tempo, embora se esperasse que este fosse compatível com o propósito da tarefa; de que poderiam utilizar quaisquer meios de consulta na internet, como dicionários e enciclopédias; e de que deveriam produzir um texto visando a sua publicação em algum periódico de circulação nacional.

No escopo dessas orientações, realizaram a tarefa do experimento no ambiente do programa Translog 2006(C, o qual registra os procedimentos realizados no teclado em tempo real. Ademais, como métodos de coleta de dados, utilizaram-se, com o auxílio do Tobii T60 e sua suíte de aplicativos, o rastreamento ocular e a gravação em vídeo dos procedimentos realizados. Ao término da tarefa, procedeu-se, ainda, à gravação de protocolos retrospectivos semiguiados.

Com base nos dados coletados nesse experimento-piloto, este trabalho buscou investigar processos de desmetaforização gramatical em instâncias que demandam esforço para serem processadas, a partir da observação de pausas e movimentos recursivos de produção textual. Para tanto, dado esse objetivo e o caráter exploratório da proposta, foram analisados os dados de dois estudantes de tradução que fizeram a pós-edição a partir do Systran e, para fins de comparação, os dados de um outro estudante de tradução que fez a pós-edição a partir do Google Tradutor. Os estudantes não foram informados sobre qual sistema de TA havia sido utilizado na tradução.

Sendo uma análise exploratória com o intuito de descrever o processo logogenético de produção textual, identificando-se Unidades de Tradução e Unidades de Alinhamento ( $c f$. ALVES et al, 2010), foram analisados os dados gerados pelos protocolos do Translog( 2006, observando-se, de forma mais pontual, as pausas e os movimentos recursivos em referência à manchete da notícia. 


\section{Análise e discussão dos resultados}

Em estudos sobre a tradução e a pós-edição, as pausas na produção textual e os processos recursivos são apontados como indicativos de esforço cognitivo ( $c f$. KRINGS, 2001; O`BRIEN, 2006; ALVES et al, 2010). Com o intuito de se investigar esse esforço em relação a processos de desmetaforização gramatical resultantes do entendimento do texto original e da tradução automática crua ( $c f$. STEINER, 2005), este estudo exploratório tomou como unidade de análise a manchete da notícia utilizada no experimento-piloto, e o processo de pós-edição dos sujeitos S1, $\mathbf{S} 2$ e $\mathbf{S 3}$, sendo que, enquanto o primeiro (S1) pós-editou a partir da tradução automática crua do Google Tradutor, os dois últimos (S2 e S3) pós-editaram a partir da tradução processada pelo Systran.

Mapeando-se as unidades de alinhamento da manchete do texto original em relação às traduções automáticas cruas, a partir da consideração da escala de ordem de grupos, frases e orações, e da função gramatical de seus constituintes, tem-se a seguinte descrição (ver QUADRO 1):

QUADRO 1 - Descrição linguistica das unidades de alinhamento do texto original em relação às traduções automáticas cruas do Google Tradutor e do Systran

\begin{tabular}{|c|c|c|c|c|c|c|c|}
\hline \multicolumn{2}{|l|}{ TO } & They & brought & the nation & \multicolumn{2}{|c|}{$\begin{array}{l}\text { to the brink of } \\
\text { default }\end{array}$} & over spending \\
\hline \multicolumn{2}{|l|}{ GT } & Eles & $\begin{array}{l}\text { trouxera } \\
\mathrm{m}\end{array}$ & a nação & \multicolumn{2}{|c|}{$\begin{array}{l}\text { à beira da } \\
\text { inadimplência }\end{array}$} & sobre os gastos \\
\hline \multicolumn{2}{|l|}{ SYS } & & $\begin{array}{l}\text { Trouxera } \\
\mathrm{m}\end{array}$ & a nação & \multicolumn{2}{|c|}{ ao limiar do defeito } & sobre a despesa \\
\hline \multicolumn{2}{|c|}{ Função } & $\begin{array}{l}\text { Participan } \\
\text { te }\end{array}$ & Processo & $\begin{array}{l}\text { Participan } \\
\text { te }\end{array}$ & \multicolumn{2}{|c|}{ Circunstância } & Circunstância \\
\hline \multirow{2}{*}{$\begin{array}{l}\text { Orde } \\
\text { m }\end{array}$} & \multirow{2}{*}{$\begin{array}{l}\text { TO } \\
\text { GT } \\
\text { SYS }\end{array}$} & $\begin{array}{l}\text { Grupo } \\
\text { Nominal }\end{array}$ & $\begin{array}{l}\text { Grupo } \\
\text { verbal }\end{array}$ & $\begin{array}{l}\text { Grupo } \\
\text { Nominal }\end{array}$ & \multicolumn{2}{|c|}{$\begin{array}{l}\text { Frase } \\
\text { preposicionada }\end{array}$} & $\begin{array}{l}\text { Frase } \\
\text { preposicionada }\end{array}$ \\
\hline & & \multicolumn{6}{|c|}{ Oração [em parataxe] } \\
\hline \multicolumn{2}{|l|}{ TO } & but & \multicolumn{3}{|c|}{ a Newsweek investigation } & \multicolumn{2}{|l|}{ shows } \\
\hline \multicolumn{2}{|l|}{ GT } & mas & $\begin{array}{l}\text { uma } \\
\text { investigação }\end{array}$ & \multicolumn{2}{|c|}{ Newsweek } & mostra & \\
\hline \multicolumn{2}{|l|}{ SYS } & mas & \multicolumn{3}{|c|}{$\begin{array}{l}\text { os legisladores de um tea party } \\
\text { das mostras da investigação de } \\
\text { Newsweek }\end{array}$} & & \\
\hline
\end{tabular}




\begin{tabular}{|c|c|c|c|c|c|c|}
\hline \multirow{2}{*}{\multicolumn{2}{|c|}{$\begin{array}{l}\text { Função } \\
\text { Ordem }\end{array}$}} & & \multicolumn{2}{|l|}{ Participante } & \multicolumn{2}{|l|}{ Processo } \\
\hline & & $\begin{array}{l}\text { Conjunçã } \\
\mathrm{o}\end{array}$ & \begin{tabular}{|l} 
Grupo \\
Nominal
\end{tabular} & $\begin{array}{l}\text { Grupo } \\
\text { Nominal }\end{array}$ & \multicolumn{2}{|c|}{ Grupo Verbal } \\
\hline \multirow{2}{*}{$\begin{array}{l}\text { Orde } \\
\text { m }\end{array}$} & $\begin{array}{l}\text { TO } \\
\text { GT }\end{array}$ & \multicolumn{5}{|c|}{ Oração paratática projetante } \\
\hline & SYS & \multicolumn{5}{|c|}{ Conjunção + grupo nominal } \\
\hline \multicolumn{2}{|l|}{ TO } & & $\begin{array}{l}\text { Tea Party } \\
\text { lawmakers }\end{array}$ & grabbing & billions & $\begin{array}{l}\text { from the government } \\
\text { trough. }\end{array}$ \\
\hline \multicolumn{2}{|l|}{ GT } & & $\begin{array}{l}\text { legisladores Tea } \\
\text { Party }\end{array}$ & agarrando & bilhões & da calha do governo. \\
\hline \multicolumn{2}{|l|}{ SYS } & que & & agarram & biliões & da calha do governo. \\
\hline \multicolumn{2}{|c|}{ Função } & & Participante & Processo & Participante & Circunstância \\
\hline \multicolumn{2}{|c|}{ Ordem } & $\begin{array}{l}\text { Conjunç } \\
\text { ão }\end{array}$ & Grupo Nominal & $\begin{array}{l}\text { Grupo } \\
\text { Verbal }\end{array}$ & $\begin{array}{l}\text { Grupo } \\
\text { Nominal }\end{array}$ & Frase preposicionada \\
\hline \multirow{2}{*}{$\begin{array}{l}\text { Orde } \\
\text { m }\end{array}$} & \begin{tabular}{|l|} 
TO \\
GT
\end{tabular} & \multicolumn{5}{|c|}{ Oração hipotática projetada (não finita) } \\
\hline & SYS & \multicolumn{5}{|c|}{ Oração hipotática } \\
\hline
\end{tabular}

A partir dessa descrição, verifica-se que, em termos estruturais e funcionais, a tradução automática crua do Google Tradutor assemelha-se mais ao texto original (TO) do que a do Systran (SYS). Com efeito, deveria apresentar um menor grau de esforço para ser pós-editada, haja vista que, seguindo o protocolo da pós-edição, haveria um menor número de modificações a serem implementadas.

Observando-se os dados do protocolo do Translog, verifica-se que S1 gastou menos tempo do que S2 e S3 para pós-editar a manchete da notícia, sendo que esse tempo foi calculado como a soma do tempo de pausas, movimentos de cursor, exclusão e produção textual no segmento (ver TABELA 1).

TABELA 1 - Tempo dispendido no segmento

\begin{tabular}{|l|c|c|c|}
\cline { 2 - 4 } \multicolumn{1}{c|}{} & $\mathrm{S} 1$ & $\mathrm{~S} 2$ & $\mathrm{~S} 3$ \\
\hline Tempo total da tarefa & $1 \mathrm{~h} 45 \mathrm{~min} 05 \mathrm{seg}$ & $1 \mathrm{~h} 42 \mathrm{~min} 26 \mathrm{seg}$ & $1 \mathrm{~h} 28 \mathrm{~min} 23 \mathrm{seg}$ \\
\hline $\begin{array}{l}\text { Tempo total dispendido } \\
\text { no segmento }\end{array}$ & $\begin{array}{c}9 \mathrm{~min} 36 \mathrm{seg} \\
(9,13 \% \text { do total })\end{array}$ & $\begin{array}{c}25 \mathrm{~min} 2 \mathrm{seg} \\
(24,43 \% \text { do total })\end{array}$ & $\begin{array}{c}12 \mathrm{~min} 59 \mathrm{seg} \\
(14,69 \% \text { do total })\end{array}$ \\
\hline
\end{tabular}


Ressalta-se, também, que o processo de pós-edição de ambos os sujeitos, independentemente do sistema de TA utilizado, deu-se em duas fases distintas, corroborando o trabalho de Vasconcellos (1989), que, a partir de sua experiência com a pós-edição na Pan American Health Organization, aponta que esta é geralmente concluída em duas fases: uma fase de correção geral (rough draft) e outra fase de polimento (polishing).

A primeira fase de correção geral demandou mais tempo para ser realizada, envolveu processos recursivos de produção textual e apresentou um número muito maior de modificações do que a segunda fase de polimento. Isso pode ser observado na produção dos sujeitos S1 e S2. Já na pós-edição de S3, verificou-se na fase de polimento um grande número de modificações, quando comparado aos demais sujeitos. Esse fato é representado nos dados estatísticos registrados pelo Translog, que aponta para um padrão processual bastante diferenciado de S3 (ver TABELA 2).

Tabela 2 - Dados processuais da tarefa registrados pelo Translog

\begin{tabular}{|l|c|c|c|}
\cline { 2 - 4 } \multicolumn{1}{c|}{} & S1 & S2 & S3 \\
\hline Total de eventos & 3588 & 2636 & 9796 \\
\hline Produção textual & 1199 & 1108 & 1607 \\
\hline Eliminação textual & 1026 & 223 & 1770 \\
\hline Movimentos de cursor & 1146 & 950 & 6320 \\
\hline Movimentos de mouse & 216 & 344 & 98 \\
\hline Eventos por minuto & 34,14 & 25,73 & 110,83 \\
\hline Produção por minuto & 11,41 & 10,82 & 18,18 \\
\hline
\end{tabular}

Observa-se a partir dos dados apresentados na TABELA 2 que, para além do sistema de TA utilizado, o perfil tradutório do sujeito tem um impacto significativo no padrão das modificações implementadas na pós-edição, conforme já observado em outros estudos ( $c f$. ALMEIDA e O`BRIEN, 2010).

Contudo, há um outro fator importante ao se considerarem as modificações na pós-edição, qual seja, o nível de expectativa do tradutor em relação à qualidade do produto final ( $c f$. ALLEN, 2003). A fim de melhor identificar essas modificações nas tarefas dos sujeitos, segundo as fases, observemos o QUADRO 2 a seguir. 
QUADRO 2 - Produção textual no processo de pós-edição

\begin{tabular}{|c|c|c|c|}
\hline Sujeito & \multicolumn{2}{|c|}{$\begin{array}{c}\text { Tempo } \\
\text { dispendido } \\
\text { no segmento }\end{array}$} & Produção textual no processo de pós-edição \\
\hline \multirow[t]{2}{*}{$\mathrm{S} 1$} & $\begin{array}{l}1^{\mathrm{a}} \\
\text { fase }\end{array}$ & \begin{tabular}{|c|}
$00: 13: 45$ \\
$\mathrm{a}$ \\
$00: 23: 15$ \\
$9 \min 30$ \\
$\mathrm{seg}$
\end{tabular} & 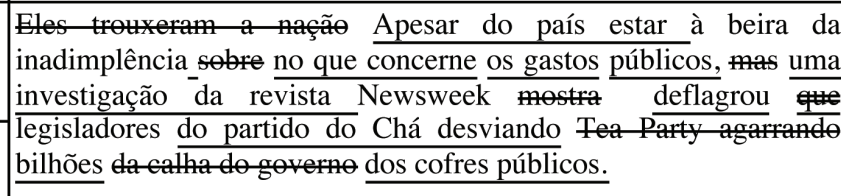 \\
\hline & $\begin{array}{c}2^{\mathrm{a}} \\
\text { fase }\end{array}$ & $\begin{array}{c}01: 37: 19 \\
\mathrm{a} \\
01: 37: 25 \\
6 \text { seo }\end{array}$ & $\begin{array}{l}\text { Apesar do país estar à beira da inadimplência com } \\
\text { os gastos públicos, uma investigação da revista Newsweek } \\
\text { deflagrou legisladores do partido do Chá desviando bilhões dos } \\
\text { cofres públicos. }\end{array}$ \\
\hline \multirow[t]{2}{*}{$\mathrm{S} 2$} & $\begin{array}{l}1^{\mathrm{a}} \\
\text { fase }\end{array}$ & $\begin{array}{c}00: 19: 23 \\
\mathrm{a} \\
00: 40: 43 \\
21 \mathrm{~min}\end{array}$ & 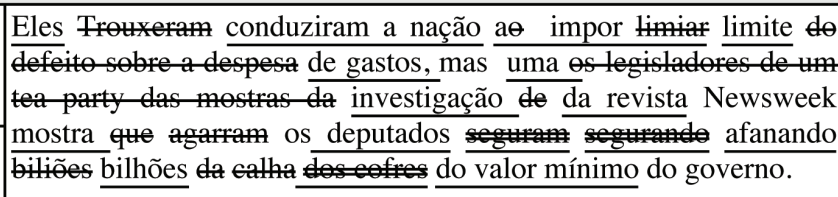 \\
\hline & $\begin{array}{c}2^{\mathrm{a}} \\
\text { fase }\end{array}$ & $\begin{array}{c}01: 34: 30 \\
\text { a } \\
01: 38: 12 \\
3 \min 42 \\
\text { seg }\end{array}$ & $\begin{array}{l}\text { Eles conduziram a nação a impor limite de gastos, mas uma } \\
\text { investigação da revista Newsweek mostra os deputados da Tea } \\
\text { Party afanando bilhões do cocho do governo. }\end{array}$ \\
\hline \multirow[t]{2}{*}{ S3 } & $\begin{array}{l}1^{\mathrm{a}} \\
\text { fase }\end{array}$ & \begin{tabular}{|c|}
$00: 10: 06$ \\
$a$ \\
$00: 17: 26$ \\
7 min 20 \\
seg
\end{tabular} & 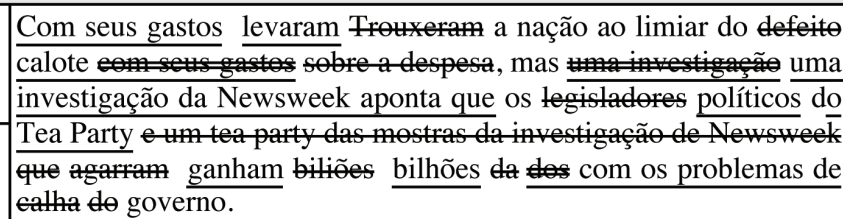 \\
\hline & $\begin{array}{c}2^{\mathrm{a}} \\
\text { fase }\end{array}$ & \begin{tabular}{c|}
$00: 46: 45$ \\
$\mathrm{a}$ \\
$00: 52: 24$ \\
$5 \min 39$ \\
$\operatorname{seg}$
\end{tabular} & 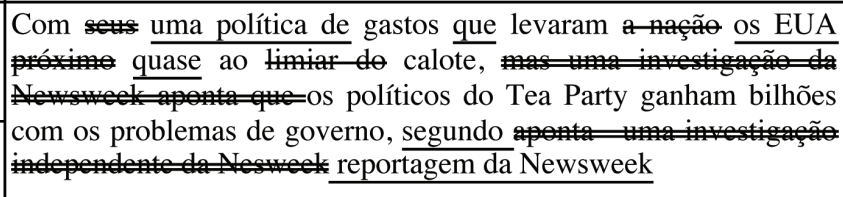 \\
\hline
\end{tabular}

O QUADRO 2 apresenta, de forma linear, a produção textual no processo de pós-edição dos sujeitos S1, S2 e S3 nas fases de correção geral e de polimento. Salienta-se, entretanto, que, embora representado de forma linear, o pro- 
cesso de produção textual em cada fase envolveu movimentos recursivos não identificados nessa representação da tarefa.

Tomando-se esse segmento, de forma arbitrária, como uma macrounidade de tradução, observa-se que os sujeitos, inclusive S1, afastaram-se do protocolo de pós-edição de implementar um número mínimo de modificações, possivelmente, motivados por um grau de expectativa elevado quanto à qualidade do produto final.

Contudo, é importante salientar que a expectativa dos tradutores quanto à qualidade do texto final passa por um processo de entendimento dos significados ideacionais, interpessoais e textuais realizados. Nesse caso, ao se alinharem os produtos finais da pós-edição dos sujeitos (S1, S2 e S3) com o texto original e as respectivas traduções automáticas cruas, verifica-se que o entendimento de algumas instâncias motivou modificações resultantes em representações da experiência com pouca correspondência semântica frente ao texto original, ou seja, houve um esforço, gerado a partir de uma expectativa, que não resultou, necessariamente, em uma representação semântica mais adequada (ver QUADRO 3).

QUADRO 3 - Unidades de alinhamento

\begin{tabular}{|l|l|}
\hline Fonte & \multicolumn{1}{|c|}{ Alinhamento do complexo oracional } \\
\hline TO & $\begin{array}{l}\text { They brought the nation to the brink of default over spending, but a Newsweek } \\
\text { investigation shows Tea Party lawmakers grabbing billions from the government } \\
\text { trough }\end{array}$ \\
\hline SYS & $\begin{array}{l}\text { Trouxeram a nação ao limiar do defeito sobre a despesa, mas os legisladores de um } \\
\text { tea party das mostras da investigação de Newsweek que agarram biliões da calha do } \\
\text { governo. }\end{array}$ \\
\hline GT & $\begin{array}{l}\text { Eles trouxeram a nação à beira da inadimplência sobre os gastos, mas uma } \\
\text { investigação Newsweek mostra legisladores Tea Party agarrando bilhões da calha } \\
\text { do governo. }\end{array}$ \\
\hline S1 & $\begin{array}{l}\text { Apesar do país estar à beira da inadimplência com os gastos públicos, uma } \\
\text { investigação da revista Newsweek deflagrou os legisladores do Partido do Chá } \\
\text { desviando bilhões dos cofres do governo }\end{array}$ \\
\hline S2 & $\begin{array}{l}\text { Eles conduziram a nação a impor limite de gastos, mas uma investigação da revista } \\
\text { Newsweek mostra os deputados da Tea Party afanando bilhões do cocho do } \\
\text { governo }\end{array}$ \\
\hline S3 & $\begin{array}{l}\text { Com uma política de gastos que levaram os EUA quase ao calote, os políticos do } \\
\text { Tea Party ganham bilhões com os problemas de governo, segundo reportagem da } \\
\text { Newsweek }\end{array}$ \\
\hline
\end{tabular}


No segmento selecionado, verifica-se que essas instâncias que demandaram esforço reverberam sobremodo em duas realizações gramaticalmente metafóricas, nomeadamente:

(i) to the brink of default over spending.

(ii) Tea Party lawmakers grabbing billions from the government trough.

No primeiro caso, conforme descrito, há duas circunstâncias, realizadas por frases preposicionadas. Em relação a essa instância, verifica-se que houve um esforço para se entender o tipo de relação circunstancial expresso por over em over spending, a qual, embora seja causal, indicando motivo (cause:reason), foi interpretada por S1 e S3 como circunstância de modo, conforme se observa no produto destes sujeitos - com os gastos públicos e com uma política de gastos, respectivamente. Ademais, ao realizarem essa relação circunstancial enquanto modo:meio (manner:means), reconfiguraram a estrutura temática da oração. Já S2 optou por editar as duas circunstâncias como uma oração não finita - $a$ impor limite de gastos, neste caso, eliminando também a identidade causal da circunstância.

No segundo caso, verificou-se um esforço para se entender o significado do processo grabbing e da circunstância from the government trough, em grabbing billions from the government trough. S1 opta por pós-editar esse segmento como - desviando bilhôes dos cofres do governo. S2, por sua vez, pósedita como - afanando bilhões do cocho do governo. Já S3 opta por - ganham bilhões com os problemas de governo. Chama a atenção que, apesar do esforço dispendido nesse segmento, o entendimento dos sujeitos resultou sobremodo em modificações lexicais (i.e. na ordem da palavra), não havendo um processo de desmetaforização gramatical que explicitasse, de forma mais congruente, os significados do texto original. Ressalta-se, ainda, que no co-texto da notícia, as opções lexicais utilizadas - desviando, afanando e ganham - produzem efeitos contextuais divergentes do texto original.

\section{Considerações finais}

Baseando-se nos dados de um experimento-piloto, este estudo explo- 
ratório buscou investigar o esforço dispendido por três estudantes de tradução em uma tarefa de pós-edição, por meio do mapeamento de pausas e processos recursivos de produção textual. Buscou-se descrever os significados produzidos, analisando-se unidades de alinhamento e de tradução referentes a uma manchete sobre a crise de gastos nos Estados Unidos, e propondo-se uma discussão entre essas unidades e o processo de (des)metaforização gramatical, resultante do entendimento dos significados ideacionais, interpessoais e textuais realizados.

Observou-se que, em termos estruturais e funcionais, a tradução automática crua do Google Tradutor (GT) assemelhava-se mais ao texto original (TO) do que a do Systran (SYS), e demandou um menor tempo para ser pós-editada. Verificou-se, também, que a pós-edição da manchete foi realizada em duas fases por ambos os sujeitos, uma de correção geral e outra de polimento, sendo que, ao contrário dos outros sujeitos, $\mathrm{S} 3$ fez um grande número de modificações na fase de polimento, o que condiz com seu perfil recursivo mapeado pelos dados estatísticos registrados pelo Translog.

Observou-se, também, que, embora a tradução automática do Google Tradutor apresentasse maior correspondência com o texto original, ambos os sujeitos implementaram um grande número de modificações durante a pós-edição, possivelmente motivados por uma expectativa elevada acerca da qualidade do produto final. Buscou-se, por sua vez, correlacionar essa expectativa com o entendimento dos significados ideacionais, interpessoais e textuais, resultante em processos de (des)metaforização gramatical. Nesse contexto, observou-se que não há uma relação direta entre o esforço dispendido na tarefa e a adequação dos significados produzidos.

\section{Referências}

ALLEN, Jeffrey. Post-editing. In: SOMERS, Harold (ed). Computers and Trans-

lation: A translator's guide. Amsterdam-Philadelphia: John Benjamins, 2003. p. 297-318.

ALMEIDA, Giselle; O'BRIEN, Sharon. Analysing post-editing performance: correlations with years of translation equivalence. In: EAMT, 2010: European As- 
sociation for Machine ranslation, St Raphael, France, 2010.

ALVES, Fábio; VALE, Daniel. Probing the unit of translation in time: Aspects of the design and development of a web application for storing, annotating, and querying translation process data. In: Across Languages and Cultures 10:1, p. 251 - 273, 2009.

ALVES, F; PAGANO, A.; NEUMANN, S. ; STEINER, E. ; HANSEN-SCHIRRA, S. . Translation units and grammatical shifts: Towards an integration of product- and process-based translation research. In: Gregory Shreve; Erik Angelone. (Org.). Translation and Cognition. Amsterdam: John Benjamins, 2010, v. 1, p. 109-142. ALVES, F. PAGANO, A.; da SILVA, I. L. Modelling (Un)Packing of Meaning in Translation: Insights from Effortful Text Production. In: Sharp et al. (eds). Proocedings of the 8th International NLPCS Workshop. Conpenhagen: Conpenhagen Business School, 2011. p.153-163.

ALVES, Fábio; VALE, Daniel. Drafting and revision in translation: a corpus linguistics oriented analysis of translation process data. In: Translation: Computation, Corpora, Cognition 1, special volume edited by Stella Neumann, Silvia Hansen-Schirra and Oliver Culo, 2011.

BAR-HILLEL, Yehoshua. The present status of automatic translation of languages. Advances in Computers, v. 1, n. 1, p. 91-163, 1960.

CARL, Michael. et al. The process of post-editing: a pilot study. In: Sharp et al. (eds). Proocedings of the 8th International NLPCS Workshop. Conpenhagen: Conpenhagen Business School, 2011. p.131-142.

CARL, Michael. Triangulating product and process data: quantifying alignment units with keystroke data. In: Copenhagen Studies in Language, 38, p 225-247, 2009.

CATFORD, J.C. A linguistic Theory of Translation. London: Oxford University Press, 1965.

GASPARI, Federico. The role of online MT in webpage translation. Faculty of Humanities: University of Manchester, 2007. 315 p. (PhD Thesis).

HALLIDAY, MAK. Things and relations: regrammaticising experience as technical knowledge. In: MARTIN, J., VEEL, R. (Ed.). Reading science; critical and functional perspectives on discourses of science. London and New York: Rout- 
ledge, 1998. p. 185-235.

HALLIDAY, M.A.K.; MATTHIESSEN, M.I.M. Christian. Construing experience through meaning: a language-based approach to cognition. London: Cassell, 1999.

HALLIDAY, M.A.K.; MATHIESSEN, Christian. An introduction to functional grammar. 3. ed. London: Edward Arnold, 2004.

HANSEN-SCHIRRA, S. NEUMANN, S., STEINER, E. Cohesive Explicitness and Explicitation in an English-German Translation Corpus. In: Languages in Contrast 7(2), p. 241-265, 2007.

HERKE-COUCHMAN, Maria. SFL, Corpus and the Consumer: An exploration of theoretical and technologial potential. Sydney: Department of Linguistics of Macquarie University, 2006. (Tese, PhD. Linguística).

HUTCHINS, John. Machine Translation: History. In: BROWN, Keith Brown (ed). Encyclopedia of Language $\boldsymbol{\&}$ Linguistics, 2 ed, v. 7. Oxford: Elsevier, 2006. p. $375-383$.

KOBY, G.S. Introduction. In: KRINGS, Hans. Repairing Texts: Empirical Investigations of Machine Translation Post-Editing Processes. Translated and edited by G.S. Koby. Kent, Ohio: The Kent State University Press, 2001.

KRINGS, Hans. Repairing Texts: Empirical Investigations of Machine Translation Post-Editing Processes. Translated and edited by G.S. Koby. Kent, Ohio: The Kent State University Press, 2001.

LOFFLER-LAURIAN, Anne-Marie (1984), Machine Translation: What Type of Post-Editing on What Type of Documents for What Type of Users? In: Proceedings of the 10th International Conference on Computational Linguistics and the 22nd Annual Meeting of the Association for Computational Linguistics. California: Stanford University, 1984. p. 236-238.

MARTÍNEZ, Lorena Guerra. Human Translation versus Machine Translation and Full Post-Editing of Raw Machine Translation Output. School of Applied Linguistics: Dublin City University, 2003. 135 p. (Master dissertation in Translation Studies).

MARTINS, Ronaldo Teixeira. Tradução automática. In: Todas as Letras, v 10, n. 2, São Paulo, 2008. p. 148-169. 
MATHIESSEN, Christian M.I.M. The environments of translation. In: Steiner, E., YALLOP, C. (Ed.). Exploring translation and multilingual text production: beyond content. Berlin, New York: Mouton de Gruyer, 2001. p.41-124.

O'BRIEN, Sharon. Teaching post-editing: A proposal for course content. In: Teaching Machine Translation - the 6th International Workshop of the European Association of Machine Translation, Centre for Computational Linguistics, UMIST: Manchester, 2002. p. 99-106.

O`BRIEN, Sharon. Machine translatability and post-editing effort: how do they relate. In: Proceedings of translating and the computer 26. London: Aslib, 2004. s.p.

O`BRIEN, Sharon. Machine translatability and post-edtitting effort: an empirical study using Translog and Choice Network Analysis. School of Applied Languages and Intercultural Studies: Dublin City University, 2006. 199p. (PhD Thesis)

OFFERSGAARD, Lene et al. Domain specific MT in use. In:Proceedings of the 12th European Association for Machine Translation conference. Hamburg, Germany, 2008. p. 153-154.

RAMOS, Luciana Cecilia. Pos-editing free machine translation: from a language vendor`s perspective. In: AMTA 2010: The Ninth conference of the American Association for Machine Translation in the Americas, Denver, Colorado, 2010. 5 p. RYAN, Joann P. The Role of the translator in making an MT system work: perspective of a developer. In: VASCONCELLOS, M. (Ed.). Technology as Translation Strategy. Amsterdam/Philadelphia: John Benjamins, 1988. p.127-132. STEINER, Erich. Intralingual and interlingual versions of a text: how specific is the notion of translation. In: Steiner, E., YALLOP, C. . (Ed.). Exploring translation and multilingual text production: beyond content. Berlin, New York: Mouton de Gruyer, 2001. p.161-190.

STEINER, Erich. Explicitation, its lexicogrammatical realization, and its determining (independent) variables - towards an empirical and corpus-based methodology. In: SPRIK REPORTS (Reports from the Project "Languages in Contrast"), n.36. Oslo: University of Oslo, 2005. p. 1-42.

TEICH, Elke. Towards a model for the description of cross-linguistic divergence 
and commonality in translation. In: STEINER, E., YALLOP, C. . (Ed.). Exploring translation and multilingual text production: beyond content. Berlin, New York: Mouton de Gruyer, 2001. p.191-228.

VASCONCELLOS, Muriel. Post-editing On-screen: Machine Translation from Spanish into English. In: Proceedings of Translating and the Computer 8. London: Aslib, 1986a, p. 133-146.

VASCONCELLOS, Muriel. Functional Considerations in the Postediting of Machine Translated Output; Dealing with V(S)O versus SVO. In: Computers and Translation, v. 1.1, p. 21-38, 1986b.

VASCONCELLOS, Muriel. Cohesion and Coherence in the Presentation of Machine Translation Products. In: ALATIS, James. Language Teaching, Testing, and Technology: Lessons from the Past with a View Toward the Future Georgetown University Round Table (GURT) on Language and Linguistics. Washington, D.C.: Georgetown University Press, 1989. p. 89-105.

WAGNER, Elizabeth. Rapid post-editing of systran. In: Tools for the trade: Proceedings of Translating and the Computer 5. London: Aslib, 1983, p.199-213.

${ }^{1} \mathrm{O}$ coautor Cristiano Gonçalves Araújo é doutorando do POSLIN/FALE/UFMG.

${ }^{2}$ Nossa tradução para "A linguistic study of intervention in the MT product at different levels of refinement can help us to prioritize our strategies. By stratifying the types of corrections that are made, we can begin to orient postediting policy so that today's MT systems are used more effectively"(VASCONCELLOS, 1989, p.89).

${ }^{3}$ Nossa tradução para: "in an age when some systems can already translate over a million words in an hour, the time that it takes to run a translation becomes insignificant; the cost-effectiveness of the MT system must be measured largely by the effectiveness of the post-editing process" (RYAN, apud O'BRIEN, 2002, p. 100).

${ }^{4}$ Nossa tradução para: "a certain amount of confidence in one's own translation ability and technical expertise is essential for this type of work. Just because rapid post-editing yields lower-quality translation, it should not be assumed that it can be undertaken by inexperienced staff. In fact it is quite the reverse - unless the post-editor has a high level of linguistic and technical knowledge he will not be able to post-edit the raw output to 
a reasonable standard in the recommended time" (WAGNER, 1983, p.204).

${ }^{5}$ Nossa tradução para: "many of the characteristics attributed to good translators could also apply to good post-editors. (...) There is an important skill that is specific to PE: the ability to decide quickly whether a machine translated segment can be useful or whether it should be ignored" (cf. OFFERSGAARD et al apud ALMEIDA e O`BRIEN, 2010, s.p). ${ }^{6}$ Nossa tradução para: "The systemic functional term grammatical metaphor is related to what is commonly called paraphrase, covering e.g., nominalization of a process. The underlying assumption here is that there is one congruent lexico-grammatical realization of a given experiential content (e.g. a semantic process is realized by a clause, a semantic entity is realizes by a nominal group, etc.) and a number of noncongruent ones which are textually and/or interpersonally not identical with the congruent one." (TEICH, 2001, p.227).

${ }^{7} \mathrm{~A}$ proposta de se trabalhar com a mudança em tradução em uma visão estratificada da linguagem tem por marco o trabalho de Catford (1965).

${ }^{8}$ Nossa tradução para: "Although TUs are ST segments, it is by analyzing ongoing target text production that we are able to momentarily capture a TU also as a target text production segment located between pauses (non-productive intervals) that can be mapped on to a source text segment. These segments may thus be correlated with, but are not identical to, TUs. A production segment is a text extract observable in TTs or in the text production process, while translation units are identified in time by pause intervals reflecting the translator's focus of attention." (ALVES et al, 2010, p. 123-124) 
